\title{
mTORC1 maintains metabolic balance
}

\author{
Cell Research (2015) 25:1085-1086. doi:10.1038/cr.2015.107; published online 11 September 2015
}

\begin{abstract}
Anticancer therapeutics aimed at the inhibition of mTORC1 activity shift metabolism to favor the degradation of extracellular proteins. Recently Thompson and colleagues demonstrated a novel regulatory mechanism whereby mTORC1 plays a distinct role as a key regulator of metabolism depending on the envi-
\end{abstract} ronmental nutrient status.

Understanding the metabolic reprogramming of cancer cells has mainly focused on their anabolic addiction to fulfill requirements for cell proliferation. In addition to rapid growth, cancer cells utilize alternative pathways to adapt themselves to various stress conditions such as growth factor and nutrient deprivation. mTORC1 is a key regulator that integrates upstream growth factor signaling pathways and controls cell growth by facilitating protein synthesis. The activity of mTORC1 is inhibited by a lack of nutrients including amino acids.

Recently Palm et al. [1] identified a novel mechanism allowing cells to alter their metabolic pathways and to acquire nutrients through regulating mTORC1 activity. Depending on environmental nutrient conditions, mTORC 1 has a distinct role in maintaining cell growth. In contrast to canonical anabolic functions of mTORC1, when cells are exposed to nutrient-rich conditions, in particular free amino acids, this complex suppresses lysosomal degradation of extracellular proteins. Conversely, inhibition of mTORC1 activity in response to nutrient deprivation significantly enhances lysosomal degradation of extracellular proteins to generate nutrients and support cell proliferation (Figure 1).
Pancreatic ductal adenocarcinoma (PDA), an extremely lethal form of cancer that typically results from KRAS mutations, relies on glutamine as a key carbon source. One source of this metabolite is extracellular protein, and oncogenic mutant KRAS induces a unique type of endocytosis, macropinocytosis, to supply the nutrients that are needed to support cancer cell growth and survival [2]. Metabolomic studies indicate that primary PDA cells are depleted for glutamine, but accumulate essential amino acids (EAAs), a pattern that suggests a critical role for the degradation of scavenged proteins to maintain cell growth [3].

mTORC1 activity, assessed by the phosphorylation of substrates including RPS6KB1/S6K1 and RPS6/S6, suppressed by EAA-deficient conditions can be restored by albumin from the environment. Because mTORC1 reactivation by albumin is abolished by the inhibition of macropinocytosis and lysosomal degradation, catabolic degradation of albumin appears to enhance mTORC1 activity and ultimately allows the cells to proliferate even under nutrient-starved conditions by supplying EAAs from protein degradation. Although macropinocytosis can be utilized as a novel route to obtain glutamine, Palm et al. show that EEAs such as leucine, isoleucine and lysine generated from the degradation of extracellular proteins play major roles in supporting cell growth and viability during nutrient starvation. Furthermore, this study reveals that the cellular metabolic balance can be determined by distinct regulation of $\mathrm{mTORC} 1$ function dependent on specific environmental nutrient availability, in particular free amino acids versus plasma protein.

The addition of albumin to cells cultured in EAA-deficient medium induces the redistribution of $\mathrm{mTORC} 1$ from a cytosolic localization to the lysosomal membrane. This albuminmediated mTORC1 localization change is impaired by the inhibition of macropinocytosis and lysosomal degradative capacity. mTORC1 reactivation from albumin degradation also requires RRAG GTPase, a well-known amino acid sensor. These data directly indicate that the products supplied from albumin degradation that lead to the restoration of mTORC1 activity are likely EAAs.

Next, the authors also examined the effect of inhibition of mTORC1 activity on lysosomal degradation and cell proliferation during nutrient starvation. When mTORC1 activity is inhibited pharmacologically or genetically by knockdown of an essential component (RPTOR/raptor) of mTORC1, cell growth is dramatically enhanced in EAA-deficient medium only in the presence of albumin. Interestingly, mTORC1 inhibition in EAA-complete conditions causes severe suppression of cell growth, implying that mTORC1 inhibitors can be considered as anticancer therapeutics only for well-vascularized tumors, reflecting nutrient-rich conditions.

Although mTORC1 inhibition increases lysosomal degradation and shows an elevation of cell growth rate during nutrient starvation, macroautophagy activity in this condition appears to be dispensable, because knockout MEFs lacking the key autophagy proteins ULK1 and ULK2 do not show a 
Oncogenic KRAS transformation

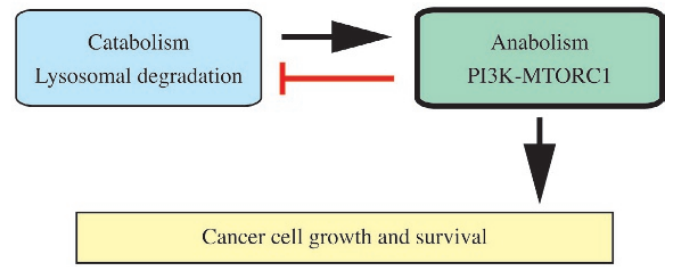

Oncogenic KRAS transformation

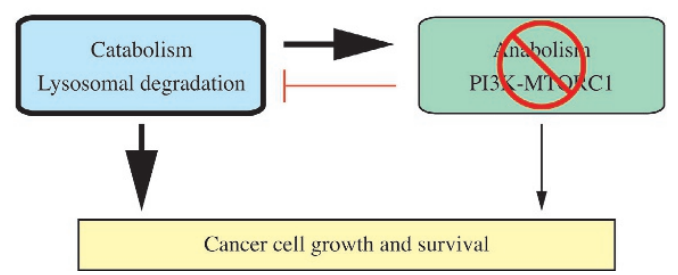

Figure 1 Schematic diagram illustrating the limitation of mTORC1 inhibitors for anticancer therapy. (A) Anabolic cellular metabolism involving the PI3K-mTORC1 pathway predominantly leads to cancer proliferation, while simultaneously suppressing catabolic cellular metabolism, which is required for cell growth and survival during nutrient starvation. This metabolic balance is finely controlled by mTORC1. (B) When $\mathrm{mTORC} 1$ is inhibited by treatment with targeted therapeutics or environmental nutrient deprivation, catabolic processes including macroautophagy and lysosomedependent degradation of extracellular proteins derived from macropinocytosis are significantly increased to support cancer cell proliferation, ultimately affecting drug efficacy depending on the tumor microenvironment.

significant defect in lysosomal degradative function and cell proliferation ability under nutrient-deprived conditions.

Finally the authors tested the effect of mTORC1 inhibition using a mouse model with pancreas-specific mutations in Kras and Trp53/p53 that lead to the development of PDA. When rapamycin, an $\mathrm{mTORC} 1$ inhibitor, is given to these mice that have an established pancreatic tumor, the proliferation rate of the tumor cells in interior and hypo-vascularized regions is dramatically increased, whereas rapamycin treatment substantially decreases the number of MKI67/ Ki-67-positive, proliferative cells in well-vascularized outer regions of the pathways could be distinguished depending on the nutrient status of the tumor microenvironment.

This work also delineates a novel molecular mechanism to understand the limitation of mTORC1 inhibitors as anticancer therapeutics, even for tumors with elevated mTORC1 activity resulting from mutation of the PI3K pathway - mTORC1 inhibitors result in the increase of a catabolic process to degrade extracellular proteins and supply them as nutrient sources in hypoxic tumors, reflecting the possibility that the tumor microenvironment might play a pivotal role in tumor progression. Moreover, RAS-mutated tumors might be more addicted to lysosomal proteolysis of extracellular proteins following macropinocytosis. Accordingly, with regard to the development of anticancer therapeutics, combinational therapy with mTORC1 inhibitors and lysosomal degradation inhibition might be expected to display synergistic efficacy.

\section{Heesun Cheong ${ }^{1}$,} Daniel J Klionsky ${ }^{2}$

${ }^{I}$ Comparative Biomedicine Research Branch, Division of Cancer Biology, National Cancer Center, 323 Ilsan-ro, Ilsandong-gu, Goyang, Gyeonggi-do 410-769, Republic of Korea; ${ }^{2}$ University of Michigan, Life Sciences Institute, Ann Arbor, MI 481098-2216, USA

Correspondence: Heesun Cheonga,

Daniel J Klionsky ${ }^{\mathrm{b}}$

aE-mail: heesunch@ncc.re.kr

bE-mail: klionsky@umich.edu

\section{References} active acquisition of nutrients from the degradation of extracellular proteins, which ultimately promotes proliferation of nutrient-starved cells. Thus, mTORC1 plays an opposite regulating role in cell proliferation depending on environmental nutrient availability, implying that the utilized catabolic
1 Palm W, Park Y, Wright K, et al. Cell 2015; 162:259-270.

2 Commisso C, Davidson SM, SoydanerAzeloglu RG, et al. Nature 2013; 497:633637.

3 Kamphorst JJ, Nofal M, Commisso C, et al. Cancer Res 2015; 75:544-553. 